\title{
Some new chapters of the long history of SU(3)
}

\author{
József $\mathrm{Cseh}^{1, *}$ \\ ${ }^{1}$ Institute for Nuclear Research, Hungarian Academy of Sciences, Debrecen, Pf. 51, 4001, Hungary
}

\begin{abstract}
The SU(3) symmetry of nuclear structure has its 60 'th birthday this year. In this contribution we recall some of its historical aspects, including several generalizations, furthermore, we discuss a few new features of this symmetry.
\end{abstract}

\section{Introduction}

In 1958 Elliott published two papers [1] on the application of the SU(3) group for the description of nuclear spectra. His model turned out to be very successful, and in addition it opened the way for many subsequent algebraic structure models.

In this contribution first, we review some basic features of this description, then mention a few extensions of the original concept. We also show some aspects of the $\mathrm{SU}(3)$ model, which have not been discussed so much, as follows. i) Spontaneous symmetry-breaking in the Elliott model. ii) The first steps towards the development of local gauge-invariant theory of nuclear collective motion. iii) The multichannel dynamical symmetry (MUSY), which extends the SU(3) connection (from 1958) between the shell collective and cluster models to the multi major shell problem, and in addition seems to have a considerable predictive power.

\section{Some interesting moments of the past}

\subsection{The birth of $\mathrm{SU}(3)$}

Elliott considered a Hamiltonian, consisting of a harmonic oscillator (HO) interaction and the sum of the quadrupolequadrupole two-nucleon interaction:

$$
H=H_{\mathrm{HO}}+\kappa Q Q
$$

This Hamiltonian is an SU(3) symmetry-preserving operator, therefore, its eigenvectors have good SU(3) quantum numbers $(\lambda, \mu)$, in addtition to the angular momentum $(L)$. The mathematical reason is that the Hamiltonian can be expressed in terms of the invariant operators of a single group-chain:

$$
\mathrm{U}(3) \supset \mathrm{SU}(3) \supset \mathrm{SO}(3) \text {, }
$$

as follows

$$
H=C_{\mathrm{U} 3}^{(1)}+\alpha C_{\mathrm{SU} 3}^{(2)}+\delta C_{\mathrm{SO} 3}^{(2)},
$$

\footnotetext{
*e-mail: cseh@atomki.mta.hu
}

where $C$ refers to Casimir-invariant of the group indicated as a subscript (and of the order indicated as a superscript). The eigenvalues of this Hamiltonian are:

$$
E=n \hbar \omega+\alpha\left(\lambda^{2}+\mu^{2}+\lambda \mu+3 \lambda+3 \mu\right)+\delta L(L+1) .
$$

Here $\hbar \omega$ is the energy of the HO excitation quantum, and $n$ is the number of quanta.

Within this framework Elliott described the quadrupole deformation and the collective rotation in terms of the spherical shell model. The SU(3) symmetry determines the quadrupole shape: $\lambda$ and $\mu$ are uniquely related to the $\beta$ and $\gamma$ parameters (for a detailed discussion see in Ref. [2]). A rotational band consists of shell-model states of a well-defined SU(3) symmetry, involving different $L$ values. This was the first connection between the two fundamental structure models of nuclei: the shell model and the liquid drop model.

Elliott's SU(3) model works very well for the description of the light nuclei.

In the same year Wildermuth and Kanellopoulos published a convenient formulation of the cluster model [3], which also presented a transparent relation between the shell and cluster models. In particular, they have shown that in the harmonic oscillator approximation the Hamiltonian of the two models can be rewritten into each other:

$$
H_{\mathrm{HOSM}}=H_{\mathrm{HOCM}} \text {. }
$$

Soon afterwards Bayman and Bohr [4] has reformulated this relation in terms of the SU(3) symmetry, therefore, by the end of 1958 the specific cluster states (just like the specific quadrupole bands) could be selected from the sea of the shell model states by their SU(3) symmetry. The cluster-shell connection, established in Ref. [3] for the harmonic oscillator interaction is valid also for more general Hamiltonians. In particular, energy-operators with the dynamical symmetry, Eq. (2), keep these relations intact [5]. Therefore, the basic connection among the three fundamental (shell, collective and cluster) models can be formulated (for a single-shell problem) by saying that their common intersection is given by the $\mathrm{SU}(3)$ dynamical symmetry of group-chain, Eq. (2). 


\subsection{Extensions}

Soon afterwards it turned out that the applicability of the Elliott model is rather limited; the SU(3) symmetry breaks down beyond the $s d$ shell (as well as with increasing energy). But it did such a beautiful job, where it was working, that much effort has been concentrated on exporting the nice features of its algebraic methods.

New models were invented, which are direct or indirect extensions of the Elliott model.

One important direction of the extension was the incorporation of many major shells instead of the single shell model [1]. The symplectic shell model [6] contains (any number of) $2 \hbar \omega$ major shell excitations (having the same parity), and in this way it is able to describe the electromagnetic transitions without introducing effective charge. Its simplified version is the contracted symplectic model [7], which has a simpler mathematical structure (e.g. compact groups instead of the noncompact symplectic one), and can be considered as a multi-shell microscopic background of the collective model. The cluster model also involves many major shell excitations, and when the internal structure of the cluster is described with the Elliott model, it can also be considered as its extension. It comes in two different versions. In the fully microscopic and semialgebraic formulation [8], only the basis states carry group symmetries, and effective nucleon-nucleon interactions are applied. On the other hand, in the semimicroscopic algebraic cluster model [9] both the basis sates and the physical operators carry group symmetries (therefore, group-theoretical methods are applied in the calculations), but phenomenological interactions are used, which are expressed in terms of group-generators. (The model space of the two approaches are identical.)

It is very remarkable that these three extensions of the Elliott model, i.e. the symplectic shell model, the contracted symplectic (collective) model and the cluster model have basis states characterized by the groupchain [10]

$$
\mathrm{U}_{s}(3) \otimes \mathrm{U}_{x}(3) \supset U(3) \supset \mathrm{SU}(3) \supset \mathrm{SO}(3) .
$$

Here $U_{s}(3)$ stands for the shell symmetry in case of the symplectic and contracted symplectic models, and for the shell structure of the cluster for the cluster model. $\mathrm{U}_{x}(3)$ describes the major shell excitations in the symplectic and contracted symplectic models in $2 \hbar \omega$ steps, and the relative motion in case of the cluster model in $1 \hbar \omega$ steps.

Another important direction of the extension of the SU(3) symmetry is along the axis of the mass number. Different approximate (or partial) symmetries have been invented, as illustrated by Fig. 1. Some of them are based on the truncation and/or rearrangement of the harmonic oscillator shell model scheme, like the pseudo-SU(3) [11], the quasi-SU(3) [12], or the proxy-SU(3) schemes [13]. Others are based on some general symmetry-breaking mechanism, like e.g. the quasidynamical symmetry [14], which is applicable also in case of other models and other symmetries.

A third route is defined by the models, which apply the algebraic method based on model assumptions, differ-

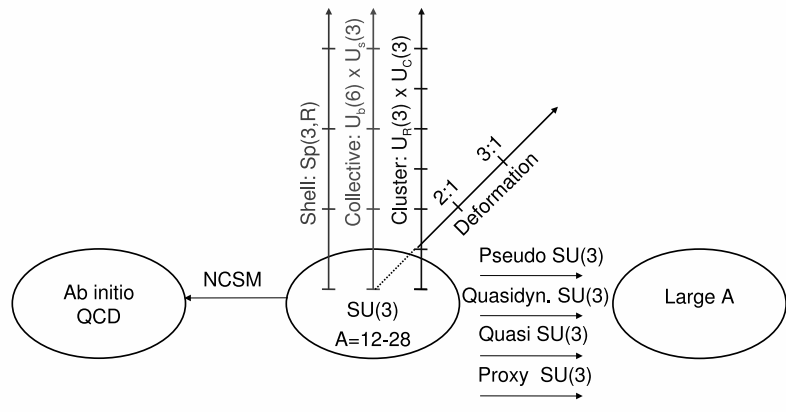

Figure 1. Some extensions of Elliott's SU(3) symmetry (central part) along different directions: mass number (horizontal) excitation energy (vertical), deformation (depth).

ent from that of the shell model. A very successful example is the interacting boson model [15], in which the basic building blocks are nucleon pairs. A further example is the quartet approach (of two protons and two neutrons) to be mentioned below.

Based on the symmetry-adapted no-core shell model approach [16] the SU(3) has recently been exported also to the territory of the very light nuclei, i.e. to the province of the ab initio methods and QCD-inspired interactions.

A further remarkable observation is that the exact SU(3) symmetry of the harmonic oscillator spherical shell model recovers for the deformed harmonic oscillator of commensurable axes [17], like, e.g., for the 2:1:1 superdeformed and for the 3:1:1 hyperdeformed shapes. For the realistic Nilsson-Hamiltonian the same kind of stability can be seen from the systematic studies based on the quasidynamical symmetry [18].

\subsection{An interesting omission}

In the history of the Elliott-model an interesting moment is related to the spontaneous symmetry-breaking. It is interesting partly because it did not appear, to the best of the knowledge of the author.

It is a frequently cited statement that the nuclear deformation is a result of the spontaneous symmetry-breaking. It has been discussed in detail in different models (see, e.g., Refs. $[15,19,20])$, but not within the Elliott-model, though it provides us with a simple and transparent framework for such an analysis.

A symmetry is spontaneously broken, if the Hamiltonian is symmetric, but its eigenstate is not invariant. Mathematically it means that the eigenvector does not transform according to the identity representation of the symmetry group. Usually the symmetry-breaking state does not even transform according to any single irrep of the symmetry group. 
The deformed ground state of an atomic nucleus with spherically symmetric Hamiltonian is a well-known example for the spontaneous breaking of a symmetry.

In the Hamiltonian, Eq. (3), of the Elliott model the last term:

$$
H_{\text {coll }}=\delta C_{\mathrm{SO} 3}^{(2)}=\delta L L,
$$

is the rotational, i.e. collective part. The first part:

$$
H_{\text {intr }}=C_{\mathrm{U} 3}^{(1)}+\alpha C_{\mathrm{SU} 3}^{(2)} \text {, }
$$

determines the bandheads, while the rotational term splits (and shifts) the bands. In this model the Hamiltonian is completely separated into an intrinsic and a collective part

$$
H=H_{\text {intr }}+H_{\text {coll }}
$$

The intrinsic Hamiltonian $H_{\text {intr }}$ is invariant under the rotation; the Casimir-operators of the $U(3)$ and SU(3) groups commute with the operators of the angular momentum (they are generators of these groups, too, and the invariant operators commute with all the generators). Nevertheless, most of their eigenstates, e.g. the ground states of many nuclei have deformed shapes. The only exceptions are the SU(3)-scalar states of $\lambda=0$ and $\mu=0$ quantum numbers, which are spherically symmetric.

Therefore, the $\mathrm{SO}(3)$ symmetry of the Schrödingerequation of the intrinsic system is spontaneously broken in many cases: a spherically symmetric Hamiltonian has a deformed eigenstate.

\section{An interesting promise of the future: gauge-theory of nuclear collectivity}

In spite of its great success, the collective model cannot describe all the collective features of the nuclei. In particular, the moment of inertia obtained from this model is too small, approximately a factor of five smaller than the experimental value. The reason is that the model describes the motion of an irrotational flow. It contains no vorticity. But the real nuclei are not liquid of irrotational flow, they correspond to an intermediate situation in between the limiting cases of the irrotational flow and rigid body.

From the microscopic viewpoint the situation is well understood. In the contracted symplectic model (which is the multi major shell microscopic picture behind the collective model), the vorticity appears due to the coupling to the valence shell structure: $\mathrm{U}_{s}(3) \otimes \mathrm{U}_{x}(3) \supset \mathrm{U}(3)$. When there is no coupling to the valence shell structure $\left(\mathrm{U}_{s}(3)\right.$ scalar case) the irrotational flow is obtained.

One can ask the question: how about the vorticity degree of freedom in the liquid drop model? Recently Rosensteel and Sparks made an interesting proposal [21]. They suggest that the vorticity can be included in the collective model, when it is transformed to a local gaugeinvariant theory.

In gauge theories the invariance of the eigenvalueequation appears due to applying two transformations simultaneously. Let us illustrate the situation with the simplest and best-known example: electromagnetism as a gauge theory. The wave function undergoes a spacedependent (local) gauge transformation: $\psi \rightarrow e^{i \alpha(x)} \psi$, and the derivative (the operator) is substituted as: $\partial \rightarrow D$, where $D=\partial+\frac{i q}{\hbar c} A$. The operator $D$ is called covariant derivative, and $q$ is the electric charge. The term arising from $A$ is cancelled through the action of $\partial$ on the phase factor. (Under the global phase transformation of the wave function: $\psi \rightarrow e^{i \alpha} \psi$, of constant $\alpha$, the equation is invariant without any changes in the operators, due to the fact that the phase factor slips through the differentiation: $\partial\left(e^{i \alpha} \psi\right)=e^{i \alpha}(\partial \psi)$. This is called global gauge invariance.) We can say that by changing the global gauge invariance into a local one the vector potential (gauge field) $A$ is introduced. The electromagnetism appears as a consequence of the requirement of the local gauge invariance. The symmetry group is the Abelian U(1), a rank-1 group, with a single generator: the electric charge [22]. In the Yang-Mills theories higher rank symmetry groups are applied [23].

In order to make the nuclear collective model gaugeinvariant, two essential ingredients are needed: the space on which the local transformation of the wave function depends on, and the transformation of the operator, i.e. the covariant derivative. (In the gauge-theories of fundamental interactions the space is the 4-dimensional spacetime.) In Ref. [21] the authors show that in case of the liquid drop model of nuclei the proper space is the 6dimensional manifold, defined by the nuclear orientation and quadrupole and monopole deformation, while the covariant derivative is:

$$
D_{\alpha}=I_{\alpha}+E_{\alpha} C_{\alpha},
$$

i.e. the angular momentum $I_{\alpha}$ is substituted by a sum of the angular momentum and a second term. The latter one contains the circulation $C_{\alpha}$, which has an $\mathrm{SO}(3)$ algebraic structure, i.e. from the mathematical viewpoint it is isomorphic to the rotational group. The coupling to circulation introduces the vorticity in the liquid drop model. (The trivial connection $E_{\alpha}=0$ determines the rigid body moment of inertia.) As a result the collective model with local gauge invariance gives the moment of inertia in agreement with experiment.

As for the future of the gauge-invariant collective model the authors have an interesting promise: by applying an SU(3) gauge instead of the $\mathrm{SO}(3)$, additional degrees of freedom are included, resulting in mixing of different circulation values within the yrast band.

\section{An interesting moment of the present: multichannel dynamical symmetry (MUSY)}

As mentioned ahead, the connection between the shell, collective and cluster models for a single-shell problem is provided by the $\mathrm{U}(3) \supset \mathrm{SU}(3) \supset \mathrm{SO}(3)$ dynamical symmetry. However, the more realistic description of the structure problems requires a multi-major-shell approach. The symplectic (shell), the contracted symplectic (collective) and the (semimicroscopic algebraic) cluster models not only 


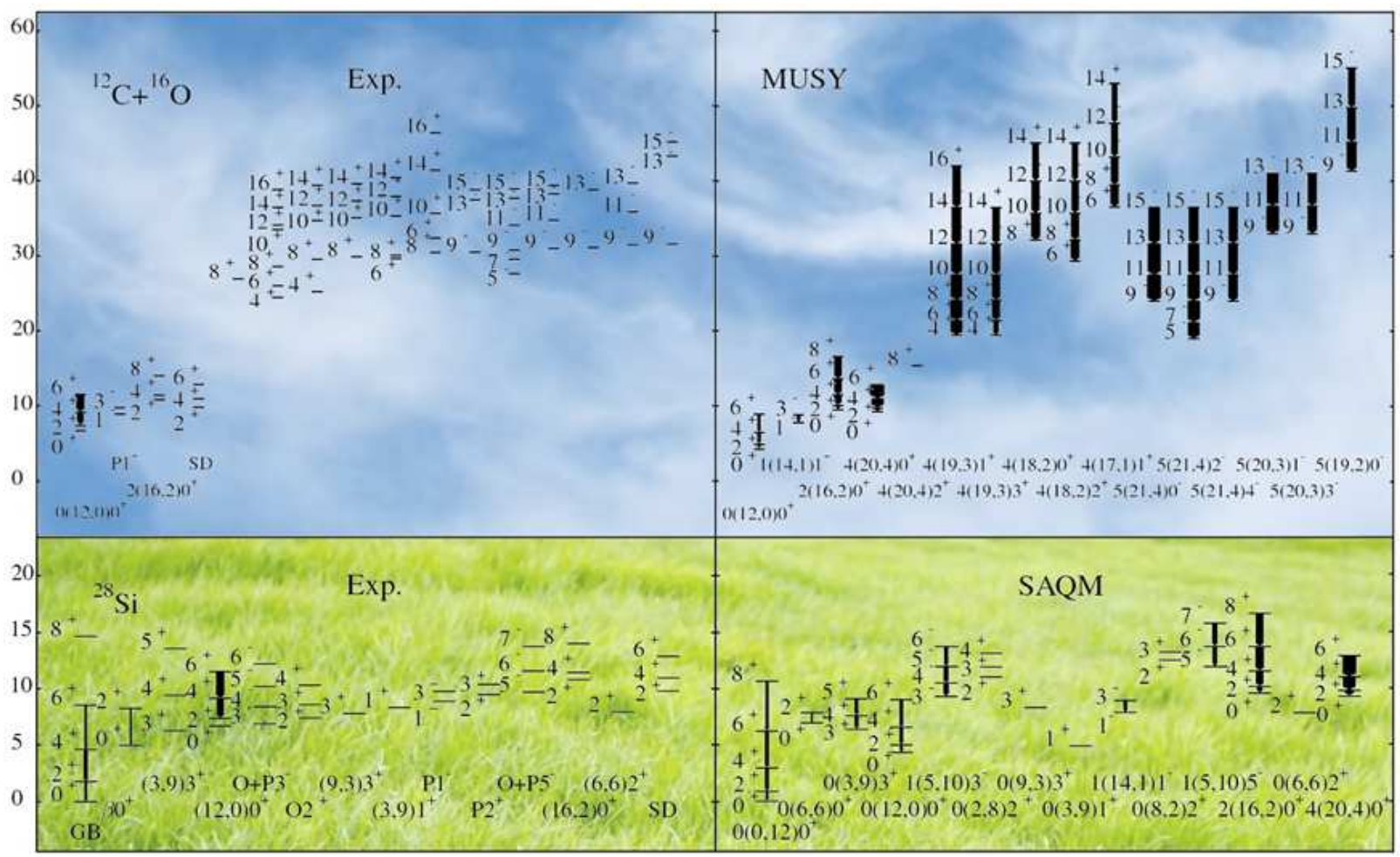

Figure 2. (Color online) Description of the low-energy bands of the ${ }^{28} \mathrm{Si}$ nucleus in terms of the semimicroscopic algebraic quartet model (lower part in the grass field), and the prediction of the MUSY for the ${ }^{12} \mathrm{C}+{ }^{16} \mathrm{O}$ cluster spectrum (upper right part in the clouds) in comparison with the experimental spectrum of the ${ }^{12} \mathrm{C}+{ }^{16} \mathrm{O}$ molecular resonances (upper left part).

offer this possibility, but they do so based on a symmetryadapted formalism, with sets of basis sates characterized by the group-chain, Eq. (6). Therefore, the common intersection of the three fundamental structure models of the multi-major-shell problem is again a dynamical symmetry [10]. In particular, the basis sates are defined by the representation labels of the groups in chain, Eq. (6), and the interactions are provided by the last part of the chain: $\mathrm{U}(3) \supset \mathrm{SU}(3) \supset \mathrm{SO}(3)$.

This symmetry was first discovered between different cluster configurations and is called multichannel dynamical symmetry (where the "channel" refers to the reaction channel which defines the cluster configuration) [24]. It was invented by requiring plausible relations between the eigenvalues of the Hamiltonians. More specifically: when the (SU(3) basis) wave functions of two different configurations have $100 \%$ overlap, i.e. they are identical (due to the effect of the antisymmetryzation), then it is natural to require that their energies should be the same.

The MUSY can incorporate the shell model configuration as well, as a special 1-cluster state. The relation has been discussed in detail in Ref. [25] in terms of the quartet model, which is a symmetry-truncated version of the no-core shell model. In particular, a quartet consists of two protons and two neutrons with $\{4\}$ permutational, and $\{1,1,1,1\}$ Wigner spin-isospin symmetry [26].

The MUSY is a composite symmetry of a composite system. The system is composite because it contains two or more different configurations. The symmetry is composite in the following sense. Each configuration has its own U(3) dynamical symmetry, and there is a further symmetry which connects them. This latter one acts in the pseudo-space of particle indices, and transforms one configuration to another one.

The formulation of the MUSY on the basis of the relations between the energy-eigenvalues is heuristic, and general, it can be applied to any configurations. However, it does not provide us with the exact mathematical details of the MUSY, e.g. the symmetry-transformations between different configurations. For the simplest case: two-channel symmetry of binary cluster configurations, the formalism is worked out in detail in Ref. [27]. It turns out that in this case the composite two-channel symmetry appears as a projection of a simple U(3) dynamical symmetry of an underlying three-cluster configuration.

The multichannel dynamical symmetry has a great predictive power. We illustrate the situation with the example of the ${ }^{28} \mathrm{Si}$ nucleus. This nucleus has a well-established band-structure in the low-energy region, and in addition good-resolution detailed spectra are known in some highlying energy windows, determined by different reactions, e.g. ${ }^{16} \mathrm{O}+{ }^{12} \mathrm{C}$ and ${ }^{24} \mathrm{Mg}+{ }^{4} \mathrm{He}$. The U(3) dynamical symmetry of the semimicroscopic algebraic quartet model has been applied for the description of the low-energy part [25]. Thus the (three) parameters of the Hamiltonian, and the (single) parameter of the $E 2$ transitions have been determined from the fit to the low-energy spectrum. Then a parameter- and ambiguity-free prediction can be made for the high-lying cluster spectra of different configurations. Figure 2 shows the situation for the ${ }^{16} \mathrm{O}+{ }^{12} \mathrm{C}$ case, 


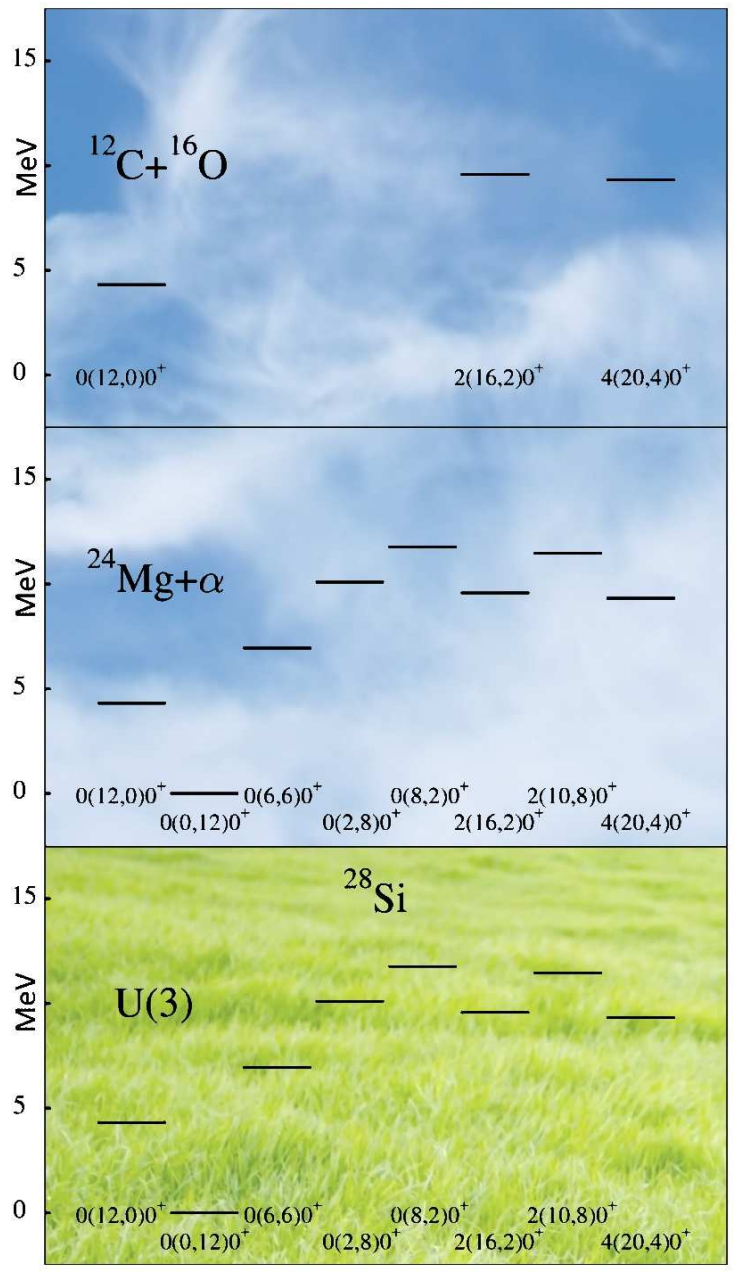

Figure 3. (Color online) The $0^{+}$spectrum of ${ }^{28} \mathrm{Si}$ nucleus from the quartet model (lower part), and the $0^{+}$states of two cluster configurations, predicted by the MUSY (upper parts).

in comparison with the spectrum of molecular resonances found in the ${ }^{16} \mathrm{O}+{ }^{12} \mathrm{C}$ experiments.

Another clusterization of considerable experimental interest is the ${ }^{24} \mathrm{Mg}+{ }^{4} \mathrm{He}$. In a recent scattering study (see, e.g., Ref. [28]) the high-lying $0^{+}$states were investigated (in order to identify the band-head of the newly-found superdeformed band [29]). This spectrum can also be predicted from the MUSY (Fig. 3), and its comparison with the experimental finding is fairly good (Fig. 4).

\section{Summary}

During its 60 years history the SU(3) symmetry did a nice job in nuclear structure studies. It described the spectrum of light nuclei, established a connection between the shell collective and cluster models, showed the way for many later algebraic structure models, served as a starting point for extensions in different directions.

In addition to its well-known features here we discussed very briefly some of its aspects which are not so frequently cited. E.g., the Elliott model illustrates nicely the spontaneous symmetry-breaking. The local SU(3) may

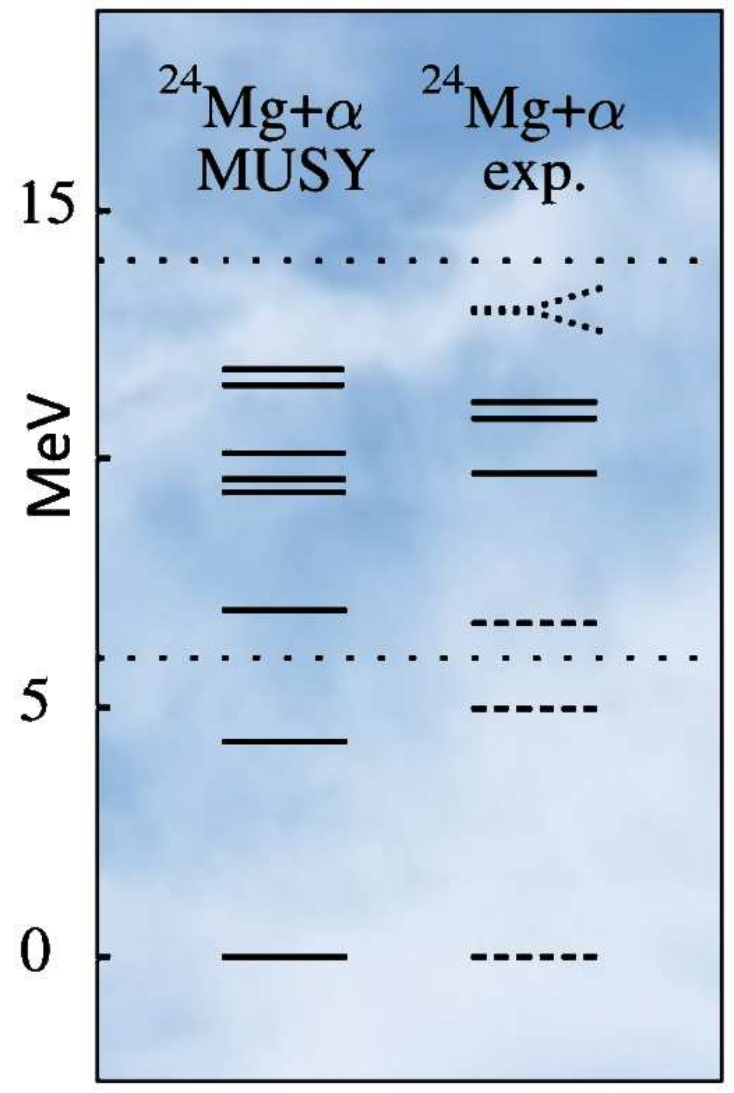

Figure 4. (Color online) Left-hand side: the $0^{+}$spectrum of the ${ }^{24} \mathrm{Mg}+{ }^{4} \mathrm{He}$ configuration, predicted by the MUSY from the quartet model description of the low-energy spectrum of ${ }^{28} \mathrm{Si}$. Righthand side: the experimental states. The energy window between the dotted lines shows the range of the inelastic alpha-scattering experiment, resulting in the states of solid lines. The states of dashed lines are from other studies.

serve as a gauge symmetry of the Yang-Mills theory of nuclear collectivity. The multichannel SU(3) symmetry connects the fundamental structure models of the many major shell problem, and seems to have a considerable predictive power. In particular, it may describe in a unified way the detailed spectra of different energy-windows and configurations, defined by the nuclear reactions.

This work was supported by the National Research, Development and Innovation Fund of Hungary, financed under the K18 funding scheme with project no. K 128729.

\section{References}

[1] J. P. Elliott, Proc. Roy. Soc. A 245, 128 (1958); Proc. Roy. Soc. A 245, 562 (1958)

[2] J. P. Draayer, in Algebraic Approaches to Nuclear Structure: Interacting Boson and Fermion Models, ed. R. F. Casten, (Harwood Academic Publishers, Singapore, 1993) p.423.

[3] K. Wildermuth, Th. Kanellopoulos, Nucl. Phys. 7, 150 (1958)

[4] B. F. Bayman, A. Bohr, Nucl. Phys. 9, 596 (1958/59) 
[5] J. Cseh, G. Lévai, A. Algora, P. O. Hess, A. Intasorn, K. Katō, Acta Phys. Hung. New Series, Heavy Ion Physics 12, 119 (2000)

[6] G. Rosensteel, D. J. Rowe, Phys. Rev. Lett. 38, 10 (1977); Ann. Phys. (N.Y.) 126, 343 (1980); D. J. Rowe, Rep. Prog. Phys. 48, 1419 (1985)

[7] G. Rosensteel, D. J. Rowe. Phys. Rev. Lett. 47, 223 (1981); O. Castaños, J. P. Draayer, Nucl. Phys. A 491, 349 (1989)

[8] H. Horiuchi, Prog. Theor. Phys. Phys. Suppl. 62, 90 (1977)

[9] J. Cseh, Phys. Lett. B 281, 173 (1992); J. Cseh, G. Lévai, Ann. Phys. (N.Y.) 230, 165 (1994)

[10] J. Cseh, J. Phys.: Conf. Ser. 580012046 (2015)

[11] R. D. Ratna Raju, J. P. Draayer, K. T. Hecht, Nucl. Phys. A 202433 (1973)

[12] A. P. Zuker, A. Poves, F. Nowacki, S. M. Lenzi, Phys. Rev. C 92, 024320 (2015)

[13] D. Bonatsos et al., Phys. Rev. C 95, 064325 (2017)

[14] P. Rochford, D. J. Rowe, Phys. Lett. B 210, 5 (1988); D. J. Rowe, P. Rochford, J. Repka, J. Math. Phys. 29, 572 (1988)

[15] F. Iachello, A. Arima, The Interacting Boson Model (Cambridge University Press, Cambridge, 1987)

[16] T. Dytrych, K. D. Sviratcheva, J. P. Draayer, C. Bahri, J. P. Vary, J. Phys. G 35, 123101 (2008)
[17] G. Rosensteel, J. P. Draayer, J. Phys. A 22, 1323 (1989)

[18] J. Cseh et al., Phys. Rev. C 80, 034320 (2009); J. Darai et al., Phys. Rev. C 84, 024302 (2011); J. Darai et al., Phys. Rev. C 86, 064309 (2012)

[19] P.-G. Reinhard, E. W. Otten, Nucl. Phys. A 420, 173 (1984); W. Nazarewicz, Int. J. Mod. Phys. E 2, 51 (1993)

[20] M. W. Kirson, A. Leviatan, Phys. Rev. Lett. 55, 2846 (1985)

[21] G. Rosensteel, N. Sparks, Europhys. Lett. 119, 62001 (2017)

[22] K. Moriyasu, An Elementary Primer for Gauge Theory (World Scientific, Singapore, 1983)

[23] K. Huang, Fundamental Forces of Nature (World Scientific, Singapore, 2007)

[24] J. Cseh, Phys. Rev. C 50, 2240 (1994)

[25] J. Cseh, G. Riczu, Phys. Lett. B 757, 312 (2016)

[26] J. Cseh, Phys. Lett. B 743, 213 (2015)

[27] J. Cseh, K. Katō, Phys. Rev. C 87, 067301 (2013)

[28] P. Adsley et al., Phys. Rev. C 95, 024319 (2017)

[29] D. G. Jenkins et al., Phys. Rev. C 86, 064308 (2012); J. Darai, J. Cseh, D. G. Jenkins, Phys. Rev. C 86, 064309 (2012) 